\title{
Trypanosoma rangeli sialidase: lack of activity in stocks from Panama and other regions
}

\author{
O ctavio E Sousa+ , Maritza Lombardo, Azael Saldaña
}

Center for Research and Diagnosis of Parasitic Diseases, Faculty of Medicine, University of Panama, Republic of Panama

A total of 33 crude and cloned Trypanosoma rangeli stocks found as natural infections in human from Panama and other endemic areas of Central and South America were evaluated as producers of sialidase (SA) activity through the MU-NANA fluorescence test. Negative results were observed in 6 of the isolates: Panama (4), Honduras (1), and Brazil (1). In addition, an immunoblotting analysis confirm the presence of the SA antigen in these stocks without enzymatic activity. These findings must be considered in the interpretation of the biological significance of T. rangeli SA and in the proper characterization and identification of this parasite.

Key words: Trypanosoma rangeli - sialidase - heterogeneity

It has been demonstrated that in some areas of Chagas disease in Central and South America there is also present a non-pathogenic trypanosome of man, Trypanosoma rangeli, Tejera 1920 (Cuba Cuba 1998, Guhl \& Vallejo 2003). This parasite has often been recovered from the blood of healthy and chagasic persons known to be infected with both parasites, $T$. cruzi and T. rangeli. Immunological studies have also shown that these parasites seem to share common protein fractions with antigenic properties which may result in some cross reactivities (Basso et al. 1989, Saldaña \& Sousa 1996). These similarities in immunogenic molecules can induce distortions in Chagas disease diagnosis and could be capable of inducing some degree of immune protection against a T. cruzi infection (Guhl et al. 1987, Sousa \& Saldaña 1994, Zuñiga et al. 1997, Palau et al. 2003).

Pereira and Moss (1985) reported on a "neuraminidase activity" present in $T$. rangeli different from a related enzyme present in T. cruzi. These authors working with stocks from El Salvador, Costa Rica, and Panama found that all strains of $T$. rangeli studied displayed neuraminidase activity and concluded that the release of the enzyme was "likely a secretory process". Further work on the T. rangeli sialidase (SA) has shown that it is not a transialidase (TSA) as in T. cruzi (Buschiazzo et al. 1993) and is prominently expressed in the epimastigote stage.

Medina-Acosta and collaborators (1994) evaluating the expression of TSA and SA activities in the Kinetoplastida Protozoa recognized four expression types: Type I, with comparable levels of TSA and SA (Endotrypanum and $T$. lewisi); Type II, expressing predominantly TSA activity ( $T$. cruzi and T. conorhini); Type III, parasites expressing SA

Financial support: SAREC/SIDA, NeTropica Program, grant 08R-2003

${ }^{+}$Corresponding author. E-mail: osousa@ ancon.up.ac.pa

Received 15 February 2005

Accepted 29 April 2005 activity exclusively (T. rangeli and T. leeuwenhoeki); and Type IV, parasites do not express either activity (Leishmania and Trypanoplasma borreli). It was considered that such interpretation permits differentiation of parasites frequent-ly found in nature (i.e. T. cruzi, T. rangeli, Leishmania/Endotrypanum).

In this study a total of 33 stocks and clones of $T$. rangeli isolated from the blood of naturally infected persons from endemic areas of Chagas disease were used to test their ability to express SA activity: from Panama (23), Guatemala (2), Honduras (3), El Salvador (1), Colombia (2), and Brazil (2). All parasites were found capable to develop in a susceptible triatomine vector host and metacyclics found in haemolymph or salivary glands.

SA activity in supernatant fluid of epimastigote cultures and whole cell lysates were measured using 2'(4methylumbelliferyl)- $\alpha$-D-N-acetylneuraminic acid (MUNANA, Sigma, St. Luis, MO) as substrate. The reaction mixture consisted of $40 \mu \mathrm{l}$ of sample and $10 \mu \mathrm{l}$ of substrate (0.25 mM MU-NANA in $100 \mathrm{mM}$ sodium acetate, $\mathrm{pH}$ 5.5). After $1 \mathrm{~h}$ incubation at $37^{\circ} \mathrm{C}$ the reaction was terminated by adding $1500 \mu \mathrm{l}$ of stop buffer $(133 \mathrm{mM}$ glycine, $84 \mathrm{mM}$ $\mathrm{Na}_{2} \mathrm{CO}_{3}, 60 \mathrm{NaCl}, \mathrm{pH} 10$ ) followed by quantification of the fluorescence emitted, with excitation at $350 \mathrm{~nm}$ and reading emission at $460 \mathrm{~nm}$ with a Hoefer TKO-100 fluorimeter. For each $T$. rangeli stock, the presence of released (Trsialr) and cellular SA (Trsials) antigen was also evaluated by immunobloting techniques with a specific polyclonal antibodies against the secreted enzyme (Saldaña et al. 1998, 2002).

The supernatant fluid of most of the 33 stocks cultures evaluated were found to exhibit strong fluorescence reactions demonstrating high SA activity. One of these, crude or cloned stocks, showed a strong positive SA reaction since its isolation from a person some 30 years ago. However, six stocks (6/33) from different regions consistently showed negative or undetectable SA activity in the MUNANA test. Four stocks from Panama, one each from Honduras and Brazil provided negative tests for SA activity both in the supernatant fluid of cultures and in cell lysates 
of the parasites (Fig. 1). The presence of the SA antigen in supernatant and cell lysate was demonstrated through immunobloting thus suggesting the presence of an inactive enzyme (Fig. 2). The present work develops information on the biological and biochemical characteristics of $T$. rangeli stocks found in natural infections in man from the Republic of Panama and other endemic areas in Central and South America and further tests the validity of the MU-NANA assay in the identification of the species. This finding calls for caution in the interpretation of the lack of SA activity in the supernatant of cell free culture media or cell lysates of $T$. rangeli stocks.

The possible evidence of spontaneous mutations in natural or laboratory culture conditions have to be considered in the evaluation of biological characterization of the parasite. This becomes of greater interest in view of recent biochemical evidence (Cremona et al. 1999, Paris et al. 2001) which suggests that $T$. rangeli SA molecule, as in $T$. cruzi TSA, is present in active and inactive forms.
Furthermore, single point mutations induced through mutagenic experiments (Buschiazzo et al. 2000) have demonstrated that single amino acid changes in the structural arrangement of the $T$. rangeli $\mathrm{SA}$ can induce strong changes in the catalytic activity of this protein.

Our results strongly suggest that $T$. rangeli stocks could be divided in two phenotypic subpopulations. Both types of parasites, SA positive and SA negative may be found affecting man in an endemic area. However, stocks showing SA activity predominate. Although the meaning of such variation remains to be investigated it appears that SA activity is not metabolically essential in some $T$. rangeli populations. Nevertheless, care must be taken not to exclude SA negative stocks when all other characters, morphological, biological, biochemical, genetic and epidemiological, are consistent with the species characteristics of $T$. rangeli. These findings also call attention to the complexity of the $T$. rangeli SA system and its biological significance.

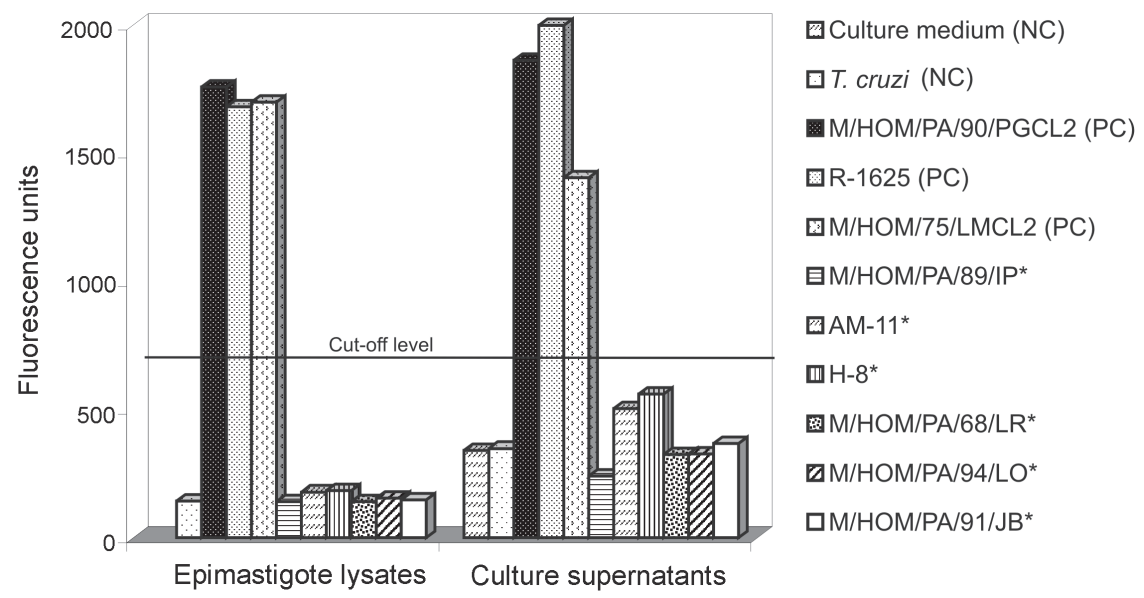

Fig. 1: detection of sialidase (SA) activity in Trypanosoma rangeli stocks. The whole cell lysates and the culture medium supernatants of different $T$. rangeli epimastigote stocks were analyzed for SA activity using 2'(4-methylumbelliferyl)- $\alpha$-D-N-acetylneuraminic acid as substrate followed by quantification of the fluorescence emitted, with excitation at $350 \mathrm{~nm}$ and reading emission at $460 \mathrm{~nm}$ with a Hoefer TKO-100 fluorimeter. Negatives (NC) and positive controls (PC) were included during each assay. Asterisks indicate the stocks without detected SA activity.

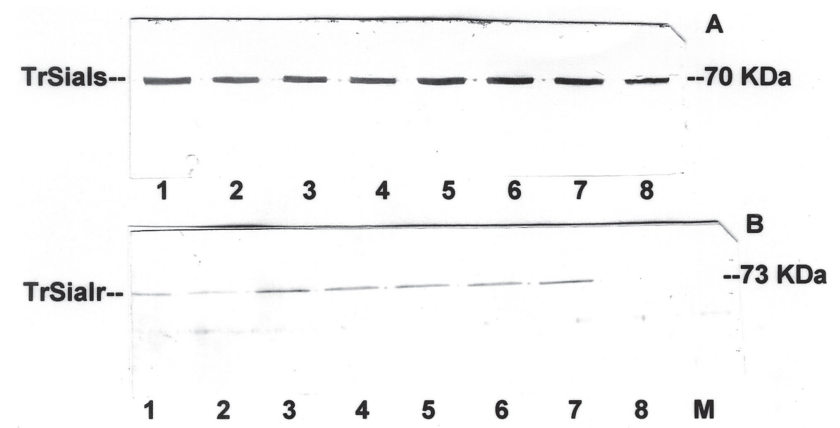

Fig. 2: antigenic expression of Trypanosoma rangeli sialidase (SA) antigen in stocks without SA activity. The whole cell lysate (A) and the medium supernatant (B) of different $T$. rangeli epimastigote stocks were analyzed for the antigenic presence of TrSials (cellular enzyme) and TrSialr (secreted enzyme) using an anti-T. rangeli SA antibody in an immunoblotting assay. The stocks identification is as follows: 1 - M/HOM/PA/94/LO; 2 - M/HOM/PA/91/JB; 3 - H-8, 4 - H/HOM/PA/68/LR; 5 - AM-11, 6 - M/HOM/PA/89/IP; 7 - M/HOM/PA/75/LM (SA activity positive control); 8 - T. cruzi IR-116B (SA activity negative control) and M (medium control). The number to the right indicate the TrSials and TrSialr molecular weights respectively. 


\section{REFERENCES}

Basso B, Moretti ER, Fontenla S, Vottero-Cima E 1989. Trypanosoma (Schizotrypanum) cruzi and Trypanosoma (Herpetosoma) rangeli. II Overlapping of antigenic spectrum. Rev Latinoam Microbiol 31: 141-146.

Buschiazzo A, Cremona ML, Campetella O, Frasch AC, Sanchez DO 1993. Sequence of a Trypanosoma rangeli gene closely related to Trypanosoma cruzi trans-sialidase. Mol Biochem Parasitol 62: 115-116.

Buschiazzo A, Tavares GA, Campetella O, Spinelli S, Cremona ML, Paris G, Amaya MF, Frasch AC, Alzari PM 2000. Structural basis of sialyltransferase activity in trypanosomal sialidases. EMBO 19: 16-24.

Cremona ML, Campetella O, Sánchez D, Frasch AC 1999. Enzymatically inactive members of the trans-sialidase family from Trypanosoma cruzi display $\beta$-galactose binding activity. Glycobiology 9: 581-587.

Cuba Cuba A 1998. Review of the biologic and diagnostic aspects of Trypanosoma (Herpetosoma) rangeli. Rev Soc Bras Med Trop 31: 207-220.

Guhl F, Vallejo GA 2003. Trypanosoma (Herpetosoma) rangeli Tejera, 1920: an updated review. Mem Inst Oswaldo Cruz 98: 435-442.

Guhl F, Hudson L, Marinkelle CJ, Jaramillo CA, Bridge D 1987. Clinical Trypanosoma rangeli infection as a complication of Chagas' disease. Parasitology 94: 475-484.

Medina-Acosta E, Franco AM, Jansen AM, Sampol M, Neves N, Pontes-de-Carvalho L, Grimaldi Junior G, Nussenzweig V 1994. Trans-sialidase and sialidase activities discriminate between morphologically indistinguishable trypanosomatids. Eur J Biochem 225: 333-339.

Palau MT, Mejia AJ, Vergara U, Zuniga CA 2003. Action of Trypanosoma rangeli in infections with virulent Trypanosoma cruzi populations. Mem Inst Oswaldo Cruz 98: 543548.

Paris G, Cremona ML, Amaya MF, Buschiazzo A, Gianbiagi S, Frasch AC, Alzari PM 2001. Probing molecular function of trypanosomal sialidases single point mutations can change substrate specificity and increase hydrolytic activity. Glycobiology 11: 305-311.

Pereira ME, Moss D 1985. Neuraminidase activity in Trypanosoma rangeli. Mol Biochem Parasitol 15: 95-103.

Saldana A, Sousa OE 1996. Trypanosoma rangeli: epimastigote immunogenicity and cross-reaction with Trypanosoma cruzi. J Parasitol 82: 363-366.

Saldana A, Sousa OE, Orn A, Harris RA 1998. Trypanosoma rangeli sialidase: kinetics of release and antigenic characterization. Acta Trop 70: 87-99.

Saldana A, Harris RA, Orn A, Monroy C, Ortega-Barria E, Sousa OE 2002. Antigenic significance of a Trypanosoma rangeli sialidase. J Parasitol 88: 697-701.

Sousa, OE, Saldaña 1994. Enfermedad de Chagas Aspectos epidemiológicos y relaciones antigénicas entre tripanosomas en areas de treansmisión por Rhodnius pallescens. Rev Medico Científica 9: 16-23.

Zuniga C, Palau T, Penin P, Gamallo C, de Diego JA 1997. Protective effect of Trypanosoma rangeli against infections with a highly virulent strain of Trypanosoma cruzi. Trop Med Int Health 2: 482-487. 
